\title{
Back-to-basics. Cough: causes and diagnosis
}

David A. Tibbutt

Retired Consultant Physician and Cardiologist, Worcestershire Royal Hospital, UK.

Correspondence:

Dr David A Tibbutt

datibb12@gmail.com

Submitted: March 2021

Accepted: June 2021

Published: August 2021
Citation:

Tibbutt, Back-to-basics. Cough: causes and diagnosis. South Sudan Medical Journal 2021;14(3):98-100 (C) 2021 The Author (s) License: This is an open access article under CC BY-NC-ND DOI:

https://dx.doi.org/10.4314/ssmj.v14i3.7

\section{Abstract}

Cough is a common complaint and may be a feature of serious underlying disease. A working knowledge of the mechanisms and differential diagnoses is crucial. A carefully taken clinical history followed by a thorough physical examination will often lead to a correct conclusion and confirmatory investigations and in turn to appropriate management.

Key words: Cough, mechanism, causes, history, examination.

\section{Introduction}

'Cough' may not seem an interesting subject to the physician but it is at best annoying to patients (and families) especially if nocturnal, and at worst very distressing particularly if associated with dyspnoea, copious sputum, haemoptysis and/or pain.

Coughing is a natural way to rid the airways of irritants and secretions, besides being a sign of a serious disease has given rise to expressions about it in Africa: "A man coughing blood is like a cow with rinderpest."

\section{Cough mechanism}

The cough mechanism is set off by the stimulation of irritant receptors, which occur in the nose and sinuses, around the vocal cords, carina and the larger airways, and also the eardrums, diaphragm, pericardium and stomach. These receptors have afferent nerves via the Vth, IXth and Xth cranial nerves to the brain. The efferent side of these reflexes leads to:

- inspiration,

- glottic closure,

- diaphragmatic relaxation,

- intercostal and abdominal muscle tension,

- $\quad$ rise of intrathoracic pressure up to $200 \mathrm{~mm} \mathrm{Hg}$.,

- glottic opening and

- invagination of the tracheobronchial membrane

- $\quad$ so narrowing the airway with

- $\quad$ rapid expulsion of air i.e., a cough

- carrying mucus with it.

In the light of this mechanism, it becomes clear why so many conditions may be associated with a cough and how the cough mechanism, which is itself often protective to the airway, may be impaired. For example, pain following abdominal surgery may reduce abdominal muscle tension, the force of a cough and so reduce mucus clearance predisposing to pulmonary infection. 


\section{Causes}

There is a useful rule to observe when considering the cause of a cough: if the cough has been present for four weeks or more always investigate for pulmonary tuberculosis. This is especially important in countries like South Sudan where tuberculosis (TB) is common.

The differential diagnosis may be reviewed from knowledge of the cough receptor sites:

\section{Upper respiratory tract:}

a. Viral infections: cough may last for months.

b. Sinusitis

c. Allergic rhinitis

d. Laryngeal lesions including inhalation of irritants.

e. Wax or any foreign body against the tympanic membrane

f. Upper airway cough syndrome (used to be called post-nasal drip syndrome).

\section{Pulmonary structures:}

a. Bronchial asthma especially in children

b. Inflammation: bronchitis, bronchiectasis, smoke irritation, migrating larval stages of parasites (e.g., hookworm, hydatid disease), malaria, $\mathrm{TB}$, lung abscess

c. Foreign bodies and tumours including Kaposi sarcoma in the immunocompromised patient.

d. Any cause of increased secretions.

e. Heart failure with pulmonary oedema, pulmonary emboli and secondary tumours.

f. Any other cause of interstitial lung disease: collagen diseases (e.g. rheumatoid disease, systemic lupus erythematosus, scleroderma), drugs (e.g. nitrofurantoin, amiodarone).

\section{Other:}

a. Diaphragmatic, pericardial and gastric receptors are probably of little importance.

b. Gastro-oesophageal reflux and either aspiration or irritation of the vocal cords

c. Psychogenic.

d. Treatment with angiotensin converting enzyme inhibitors (ACEI).

In South Sudan important causes, and types, of cough are:

1. Unilateral or bilateral pleural effusion.

2. Pneumocystis jirovecii pneumonia or Kaposi sarcoma in immuno-compromised patients, the cough is usually dry

3. Large pulmonary balls of hydatid disease.
4. Persistent cough in children is almost always a sign of bronchial asthma

5. Hydro- and pyo-pneumothorax coughing.

6. Suppurative lung abscess should include pyopneumothorax which produces pus in sputum if there is pulmonary-bronchial fistula.

Special points to note in the history

1. Times when the cough is worse: cough at night may indicate heart failure, bronchial asthma or aspiration from gastro-oesophageal reflux; cough at meal times may suggest aspiration. Being woken with paroxysmal cough is usually due to reflux.

2. Bronchial hyperreactivity: Cough triggered by dust, fumes, temperature change, cold air e.g. cough variant asthma.

\section{Triggers:}

a. Exertion or laughing: interstitial lung disease or bronchial asthma.

b. Environmental change: houses and pets: may suggest an allergic cause.

c. Medication: beta-blockers and ACEI. Interstitial lung disease may be caused by many drugs (e.g., anticancer drugs, penicillins, non-steroidal antiinflammatory drugs) and present with cough.

4. Sputum production: Post-nasal drip and bronchitis often create morning sputum. Purulent sputum usually suggests bacterial infection but eosinophils in large numbers may give rise to similar appearances. Large volumes of purulent sputum suggest bronchiectasis.

5. Haemoptysis: TB, bronchiectasis, tumours, pulmonary embolism and bronchitis.

6. Sneezing and rhinorrhoea: allergic rhinitis.

7. Dry mouth, from mouth breathing, change or loss of sense of smell: chronic rhinitis.

8. Gastro-oesophageal reflux symptoms or dysphagia: oesophageal disease. Note that symptoms of reflux may be absent if on antacid treatment, but the non-acidic refluxate may still cause cough

9. Joint pains and/or swelling: connective tissue disorders.

10. Pointers to acquired immunodeficiency: unexplained cough, often dry, may be a presentation of Pneumocystis jirovecii pneumonia or Kaposi sarcoma.

11. COVID-19 has become a major global concern and frequently presents with a persistent cough, change of sense of smell and taste, and fever. 


\section{Special points to note on examination}

1. Deep expiration precipitating the cough suggests bronchial asthma.

2. Deep inspiration precipitating the cough suggests interstitial lung disease.?

3. Types of cough:

a. "Wet": bronchial asthma, bronchitis.

b. "Brassy": tumour.

4. Increasing cough during examination and clearing when the patient is not aware of being observed may suggest a psychogenic cause.

5. Impacted ear wax.

6. Nasal passages: polyps, mucopurulent discharge, signs of inflammation.

7. Tender maxillary sinuses.

8. Goitre and other neck masses.

9. Rales and rhonchi especially if localised.

10. Finger clubbing with malignancy, lung abscess, bronchiectasis and occasionally with tuberculosis.

11. Central cyanosis and/or anaemia.

Careful clinical assessment will provide a working diagnosis in most cases and special investigations (such as those listed below) often are not needed especially where facilities are lacking.

Sputum examination: Look at it!!! Are there any signs of blood? A rusty looking specimen may suggest a pneumococcal pneumonia. Microscopy will differentiate bacterial infection from eosinophilia: consider $\mathrm{TB}$ and fungi.

Chest X-ray: A normal X-ray does not exclude tuberculosis, tumour, foreign body or bronchiectasis. In areas where it is known to occur, hydatid disease may produce large pulmonary "balls". An unsuspected (hydroor pyo-) pneumothorax may present with cough. A lung abscess associated with a broncho-pulmonary fistula gives rise to large amounts of purulent sputum.

Respiratory function tests: the simplest is to observe the patient exercising e.g., walking the length of a ward or climbing stairs. The inability to complete sentences without added inspirations during ordinary conversation is abnormal. Exercise may induce wheezing in bronchial asthma. Peak expiratory flow (PEF) measurement is helpful, but spirometry is better. PEF meters are small, relatively inexpensive and the measurements at least give some quantitative idea of progression. Without such equipment ask the patient to blow out a lighted match from five inches and with the mouth open.

\section{Treatment}

It is not the purpose of this article to describe the treatment of all the causes of cough:

1. Treatment should be directed at the specific cause.

2. Removal of an allergen or irritant is usually very effective: cigarette smoking is an important example.

3. "Bronchitis" that does not respond to antibiotics should suggest an obstructing lesion.

4. A cough associated only with sputum should not be suppressed.

5. A distressing cough with an irreversible cause (e.g., metastatic malignancy) should be suppressed using:

a. A simple linctus or

b. Humidified air (steam inhalation but care to avoid scalding) or

c. Codeine phosphate 30-60mg. 6-8 hourly (but be aware of the constipating effect)

d. Kindness and reassurance: a patient is often more afraid of the cause of the cough than the cough itself.

6. Aggressive management of oesophageal reflux may be needed because $\mathrm{H} 2$ blockers or proton pump inhibitors will not affect proteases. So, raising the head of the bed, avoiding eating late at night may be helpful.

7. Trial of inhaled steroid may help manage cough variant asthma.

Explaining to a patient the cause of the cough and its prognosis and treatment is important. In South Sudan patients are most scared if they have haemoptysis, with some believing that they will not survive for long after the episode. Many people with bronchial asthma resist nebulized treatment and need to be persuaded to accept it.

Acknowledgements: I thank Prof. Richard Lewis, retired chest physician, who helped to prepare this paper and also Prof. Peter Otto, Chest Physician, South Sudan

\section{Further reading}

1. Polverina M, Polverino F, Fasolino $M$ et al. Anatomy and neuropathophysiology of the cough reflex arc. Multidisciplinary Respiratory Medicine, 2012; 7(1):5 https://mrmjournal.biomedcentral. com/articles/10.1186/2049-6958-7-5

2. "Chronic cough". Mayo Clinic. https://www. mayoclinic.org/diseases-conditions/chroniccough/symptoms-causes/syc-20351575.

3. “Cough”. Mayo Clinic. https://www.mayoclinic. org/symptoms/cough/basics/causes/sym20050846

4. Vally M, Irhuma MOE. Management of cough: a practical approach. South African Family Practice 2016;58(4):35-39. 